\title{
3D structure of nearby groups of galaxies
}

\author{
L. Makarova ${ }^{1}$, D. Makarov ${ }^{1}$, A. Klypin ${ }^{2}$ and S. Gottlöber ${ }^{3}$ \\ ${ }^{1}$ Special Astrophysical Observatory, Nizhniy Arkhyz, Karachai-Cherkessia 369167, Russia \\ email: lidia@sao.ru \\ ${ }^{2}$ Astronomy Department, New Mexico State University, MSC 4500, P.O.Box 30001, Las \\ Cruces, NM, 880003-8001, USA \\ ${ }^{3}$ Leibniz-Institut für Astrophysik (AIP), An der Sternwarte 16, D-14482 Potsdam, Germany
}

\begin{abstract}
Using high accuracy distance estimates, we study the three-dimensional distribution of galaxies in five galaxy groups at a distance less than $5 \mathrm{Mpc}$ from the Milky Way. Due to proximity of these groups our sample of galaxies is nearly complete down to extremely small dwarf galaxies with absolute magnitudes $M_{B}=-12$. We find that the average number-density profile of the groups shows a steep power-law decline $d n / d V \sim R^{-3}$ at distances $\mathrm{R}=(100-500)$ $\mathrm{kpc}$ consistent with predictions of the standard cosmological model. We also find that there is no indication of a truncation or a cutoff in the density at the expected virial radius: the density profile extends at least to $1.5 \mathrm{Mpc}$. Vast majority of galaxies within $1.5 \mathrm{Mpc}$ radius around group centres are gas-rich star-forming galaxies. Early-type galaxies are found only in the central $\sim 300$ kpc region. Lack of dwarf spheroidal and dwarf elliptical galaxies in the field and in the outskirts of large groups is a clear indication that these galaxies experienced morphological transformation when they came close to the central region of forming galaxy group.
\end{abstract}

Keywords. (cosmology:) distance scale, (cosmology:) dark matter, galaxies: distances and redshifts, galaxies: dwarf

We use the Updated Nearby Galaxy Catalog (Karachentsev, Makarov, Kaisina, 2013, AJ, 145, 101), selecting seven nearest well-known groups of galaxies. Each of the group contains one main central galaxy and a significant family of satellite galaxies. We surveyed the groups around the Milky Way galaxy, M31 (Andromeda), M81, Centaurus A, M83, Canes Venatici I cloud (M94), and Sculptor (NGC253).

The luminosity function (LF) (the left panel of the Fig. 1) is constructed using adaptive binning with 10 galaxies per bin. The combined luminosity function does not show any indication of cut off on the bright end of the distribution. A simple power law $n \sim L^{\alpha}$ fits it well with a slope $\alpha=-1.24 \pm 0.02$. The LF of all galaxies within $7 \mathrm{Mpc}$ is fitted by a Schechter function with a slope $\alpha=-1.26 \pm 0.04$. The faint end of the LF for combined group is in good accordance with the whole $7 \mathrm{Mpc}$ sample.

The observed density profile (the right panel of the Fig. 1) does not show any statistically significant drop or any other feature around the virial radius. The virial radius for these type of galaxies is expected to be in the range of $\sim 200-400 \mathrm{kpc}$. The density profiles smoothly declines from large $(\sim 1000)$ overdensities in the central $\sim 100 \mathrm{kpc}$ region and gradually approaches the background at $\sim 1 \mathrm{Mpc}$. The dashed curve shows the Navarro-Frenk-White profile with parameters compatible with LCDM predictions: virial mass $2.5 \times 10^{12} M_{\odot}$, concentration $\mathrm{C}=10$. The virial radius for this halo is shown in the plot. The average density of the Universe is added to the Navarro-Frenk-White profile.

The distribution of galaxies in the considered groups by morphological types is demonstrated in the Fig. 2. Note, that dSphs present about $40 \%$ of the whole sample, while outside the virialized zone they consist only $20 \%$ of galaxies. It illustrates the fact that 

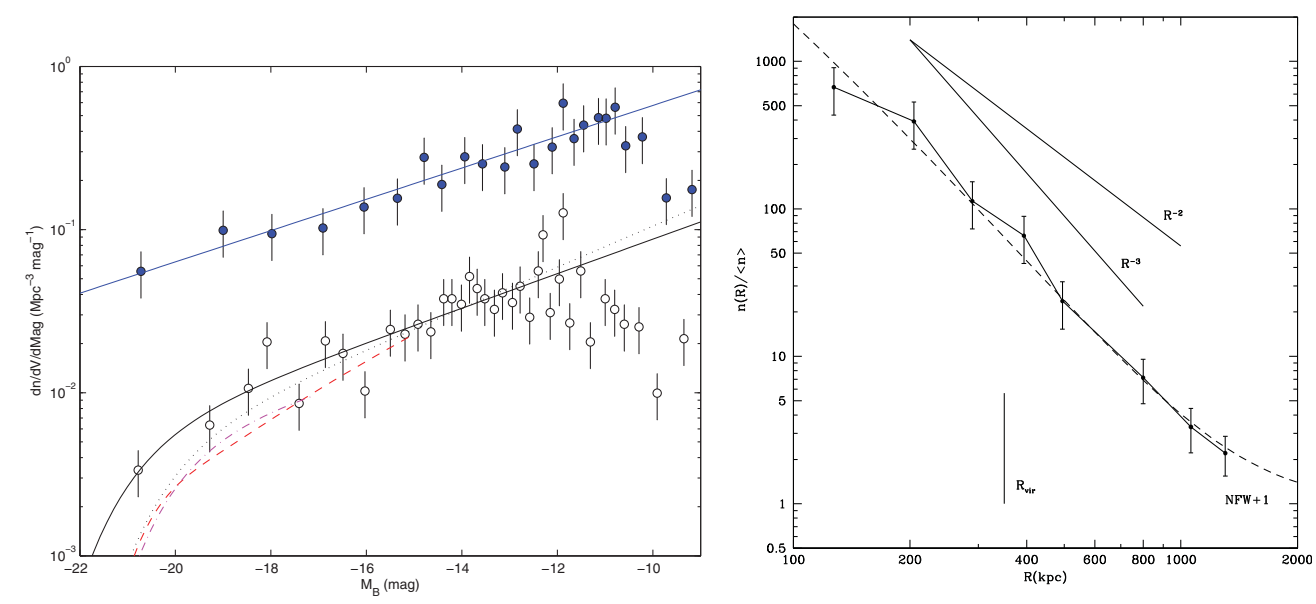

Figure 1. Left panel: the LF of the considered groups is shown with filled circles. Open symbols display the LF of all Local Volume galaxies within $7 \mathrm{Mpc}$ from UNGC. For comparison we plot the global LFs from SDSS (Blanton et al. 2005, ApJ, 631, 208) as long dashed line and from 2dFGRS (Norberg et al. 2002, MNRAS, 336, 907) as dashed-dotted line. The dotted line illustrates it using the Schechter approximation of nearby galaxies with fixed cut-off $M_{B}^{*}=20.5$, which roughly corresponds to the global value. Right panel: the full curve with error bars shows the average number-density of galaxies in four isolated centrally dominated groups: Cen A, M 83, M 81, and NGC 253. The number-density was normalized to the average number-density of galaxies brighter than $M_{B}=12$ inside $10 \mathrm{Mpc}$ region. The errors are poissonian errors.
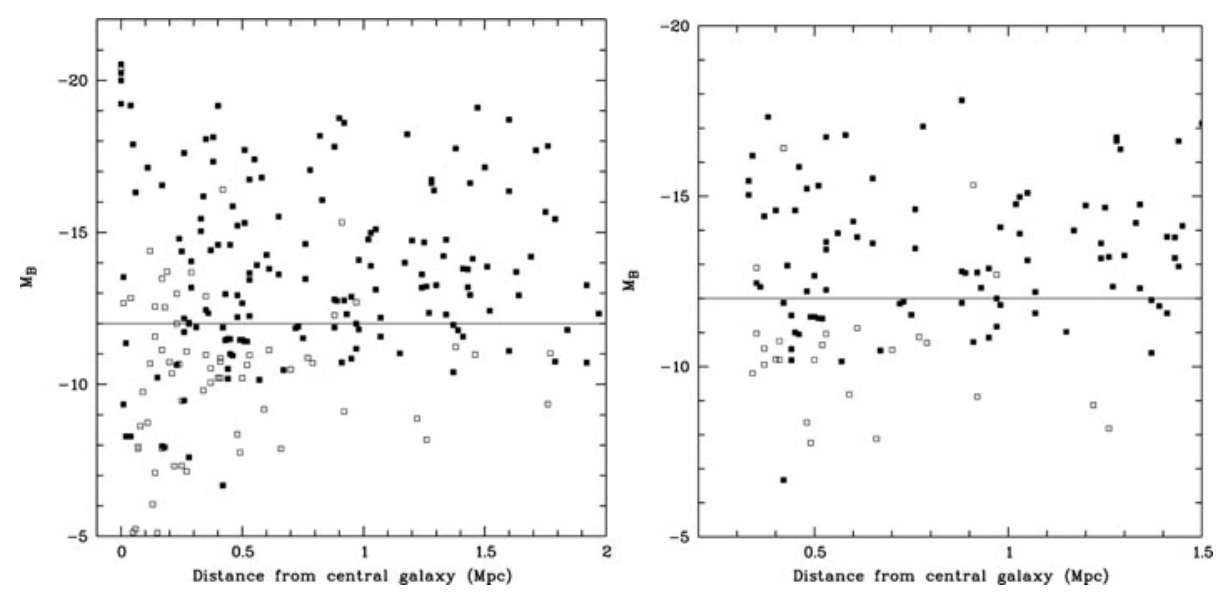

Figure 2. Left panel: the distribution of the absolute magnitudes of galaxies, corrected for galactic and internal absorption as a function of the distance of the galaxy from the central object in the group. Open squares represent giant and dwarf ellipticals and dwarf spheroidal galaxies, whereas filled squares are giant spirals, S0, irregular and dwarf irregular galaxies. Right panel: the same galaxies, but located in the non-virialized zone of each of the group, that is, $0.3<\mathrm{r} \leqslant 1.5 \mathrm{Mpc}$. The resulting subsample contains $50 \%$ of the original one.

most of dSphs located inside $0.3-0.5 \mathrm{Mpc}$ from the central galaxies, while dIrs are distributed more widely.

Acknowledgements. We acknowledge the support from RFBR grant 13-02-00780 and Research Program OFN-17 of the Division of Physics, Russian Academy of Sciences, and the grant of Russian Scientific Foundation 14-12-00965. Our collaboration has been supported by the German Science Foundation (DFG). 